ARTICLE

\title{
Asiatic acid enhances intratumor delivery and the antitumor effect of pegylated liposomal doxorubicin by reducing tumor-stroma collagen
}

\author{
Luo Fang ${ }^{1,2}$, Si-si Kong ${ }^{1}$, Li-ke Zhong ${ }^{1}$, Can-ming Wang ${ }^{1}$, Yu-jia Liu ${ }^{1}$, Hai-ying Ding ${ }^{1}$, Jiao Sun ${ }^{1}$, Yi-wen Zhang $^{1}$, Fan-zhu Li ${ }^{3}$ and \\ Ping Huang ${ }^{1}$
}

Tumor-targeted drug delivery systems (Tt-DDSs) are proposed as a promising strategy for cancer care. However, the dense collagen network in tumors stroma significantly reduces the penetration and efficacy of Tt-DDS. In order to investigate the effect of asiatic acid (AA) on antitumor effect of pegylated liposomal doxorubicin (PLD) by attenuating stroma-collagen, colon cancer xenograft mice (SW620 cell line) were treated by PLD, AA, or combined regimes, respectively; the collagen levels were estimated by Sirius red/ fast green dual staining and immunohistochemistry $(\mathrm{IHC})$ staining; the intratumor exposure of doxorubicin was visualized by ex vivo fluorescence imaging and quantified by HPLC/MS analysis. In addition, the impact of AA on collagen synthesis of fibroblast cell (HFL-1) and cytotoxic effect of PLD and doxorubicin to cancer cell (SW620) were studied in vitro. In the presence of AA (4 mg/kg), the intratumor collagen level was restricted in vivo (reduced by $22 \%$, from $4.14 \% \pm 0.30 \%$ to $3.24 \% \pm 0.25 \%, P=0.051$ ) and in vitro. Subsequently, doxorubicin level was increased by $\sim 30 \%$. The antitumor activity of PLD was significantly improved $(57.3 \%$ inhibition of tumor growth and $44 \%$ reduction in tumor weight) by AA combination. Additionally, no significant improvement in cytotoxic effect of PLD or doxorubicin induced by AA was observed. In conclusion, AA is a promising sensitizer for tumor treatment by enhancing intratumor drug exposure via stromal remodeling.

Keywords: colon cancer; asiatic acid; pegylated liposomal doxorubicin; stromal collagen; intratumor drug delivery

Acta Pharmacologica Sinica (2019) 40:539-545; https://doi.org/10.1038/s41401-018-0038-2

\section{INTRODUCTION}

Tumor-targeted drug delivery systems (Tt-DDSs) were developed as a breakthrough strategy for cancer care by improving patient benefits from cytotoxic agents [1]. However, the clinical effectiveness of Tt-DDSs was questioned because no difference in efficacy was found between the approved liposomal and conventional chemotherapies [2]. For example, pegylated liposomal doxorubicin (PLD), the first approved "steal" liposome characterized as a long-circulation and tumor-targeting liposome, reduced the risk of many side effects of doxorubicin (DOX), including cardiac toxicity, but failed to show significant superiority in clinical benefit or transfer to wide use in clinical practice compared with conventional DOX $[3,4]$.

Most recently, accumulated evidence suggested that impaired distribution of anti-neoplastic agents from vessels to cancer cells due to dense stroma is a crucial contributor to the compromised effect of anti-neoplastic agents, especially macro-molecular agents, such as Tt-DDSs [5-7]. The extracellular matrix of the tumor consists of highly compact, sheet-like collagen networks, which separate cancer cells from microvessels and limit the convection and diffusion of Tt-DDSs by increasing the interstitial fluid pressure, narrowing the matrix space, and elongating the diffusion path with a tortuous interstitial space [8-10]. Several interventions for matrix degradation, such as relaxin hormone [11, 12], matrix metalloproteinases [13], bacterial or bovine hyaluronidases [14, 15], losartan [16], and Smo inhibitor [17], were proven to enhance intratumor delivery of macro-molecular vectors. Therefore, reduction of structural collagens is proposed as a novel concept to improve chemotherapeutic effect by enhancing the permeability of Tt-DDSs from the microvessels to cancer cells.

However, most of the aforementioned interventions encounter difficulty in meeting the requirements of translational medicine for high risk of toxicity. Moreover, most previous research studies were focused on the permeability profile of nano-vectors instead of the antitumor effect of approved DDSs. Rakesh and colleagues reported a pilot result of improved efficacy for single-cycle PLD in vivo by losartan [16]. However, more convincing evidence of the stroma-modulator boosting effect of full cycles of chemotherapy with approved DDSs are still needed. Therefore, we evaluated the role of a natural source anti-desmoplastic agent, i.e., asiatic acid (AA), in full-course chemotherapy of PLD.

$A A$ is a pentacyclic triterpene isolated from the traditional Chinese medicine Centella asiatica with antioxidant, anti-inflammatory, neuro-protection, antidiabetes, and antitumor activities

\footnotetext{
${ }^{1}$ Department of Pharmacy, Zhejiang Cancer Hospital, Hangzhou 310022, China; ${ }^{2}$ Zhejiang Key Laboratory of Diagnosis and Treatment Technology on Thoracic Oncology (Lung and Esophagus), Hangzhou 310022, China and ${ }^{3}$ College of Pharmaceutical Science, Zhejiang Chinese Medical University, Hangzhou 310022, China

Correspondence: Fan-zhu Li (lifanzhu@zcmu.edu.cn) or Ping Huang (Huangping1841@zjcc.org.cn)

These authors contributed equally: Luo Fang, Si-si Kong.
}

Received: 4 December 2017 Accepted: 3 May 2018

Published online: 19 June 2018 
[18-22]. AA displayed robust inhibition of fibrosis by inhibiting collagen matrix expression [18-21]. In the current study, we investigated whether AA could alleviate over-expression of intratumor collagen, increase intratumor accumulation of PLD, and ultimately boost the antitumor effect in mice bearing xenografts of SW620 colon cancer.

\section{MATERIALS AND METHODS}

Chemicals and reagents

The pegylated liposomal DOX injection solution (Libod ${ }^{\oplus}, 10 \mathrm{~mL}$ : $20 \mathrm{mg}$ ) was supplied by Shanghai Fudan-zhangjiang Bio-Pharmaceutical (Shanghai, China). AA, DOX, paraformaldehyde, and dimethyl sulfoxide were purchased from Sigma (St Louis, MO, USA). DMEM:F12 (1:1) media and fetal bovine serum (FBS) were supplied by Thermo Fisher Scientific. Phosphate-buffered saline (PBS), trypsin-EDTA $(0.25 \%, 1 \times)$, and penicillin-streptomycin $(10000 \mathrm{U} / \mathrm{mL})$ were obtained from Solarbio Life Sciences (Beijing, China). Antibodies of collagen type I (ab34710) and IV (ab6586) were obtained from Abcam (Cambridge, UK), except for GAPDH (Mab5465), which was obtained from Hangzhou MultiSciences Biotech, Co., Ltd., Hangzhou, China. The Sirius red/fast green collagen staining kit was purchased from Chondrex (Catalog \# 9046, Redmond, USA). Other reagents were purchased from Sinopharm Chemical Reagent Co. Ltd (Shanghai, China).

Cell line culture and treatment

Human colorectal adenocarcinoma (SW620) and fibroblast (HFL-1) cell lines were obtained from Professor Peihua Luo (Zhejiang Province Key Laboratory of Anti-Cancer Drug Research, Hangzhou, China) and the Shanghai Cell Bank of the Chinese Academy of Sciences (Shanghai, China), respectively. The SW620 cells were cultured in DMEM:F12 (1:1) containing 10\% FBS, $100 \mathrm{U} / \mathrm{mL}$ streptomycin, and $100 \mathrm{U} / \mathrm{mL}$ penicillin at $37^{\circ} \mathrm{C}$ in a humidified $5 \% \mathrm{CO}_{2}$ atm. The HFL-1 cells were activated and maintained in SW620-derived conditioned medium (CM) collected as follows. The supernatant of used medium from SW620 cells at $>80 \%$ confluency was collected by centrifugation, passed through a $0.22 \mu \mathrm{m}$ filter, and diluted with DMEM:F12 (1:1) containing 20\% FBS at a ratio of 1:1. The DMEM:F12 (1:1) medium supplemented with $10 \%$ FBS served as the control medium. The HFL-1 cells were treated with varying concentrations of $A A(0.5,1$, or $2.00 \mu \mathrm{M}$, three predetermined $20 \%$ viability-inhibitory concentration of AA to HFL-1, data not shown), and the total RNA and proteins were extracted for quantitative real-time polymerase chain reaction (qRT-PCR) and western blot analysis, respectively.

\section{Cytotoxicity assay}

To determine the impact of AA on the antitumor effect of PLD in vitro, the viability profiles of SW620 treated by PLD combined with AA were estimated via CCK-8 assay according to the manufacturer's instructions. SW620 cells were seeded into 96well plates at $80 \%$ confluence, cultured for $24 \mathrm{~h}$, and additionally treated for $72 \mathrm{~h}$ with varying concentrations of PLD $(0,0.3125$, $0.625,1.25,2.5,5,10,20,40$, and $80 \mu \mathrm{M})$ or $\operatorname{DOX}(0,0.0015,0.006$, $0.024,0.098,0.39,1.56,6.25,25$, and $100 \mu \mathrm{M})$ alone or combined with varying concentrations of $A A(0.5,1.0$, or $2.0 \mu \mathrm{M})$. The absorbance at $450 \mathrm{~nm}$ was measured using a Microplate Spectrophotometer (Epoch 2, BioTek ${ }^{\circledR}$, Vermont, USA)

\section{qRT-PCR analysis}

HFL-1 cells were seeded on six-well plates and cultured for $24 \mathrm{~h}$. Total RNA was extracted from the cells using TRIzol reagent (Invitrogen $^{\mathrm{TM}}$, Carlsbad, CA, USA) $24 \mathrm{~h}$ after treatment with various concentrations of AA $(0,0.5,1.0$, or $2.0 \mu \mathrm{M})$. Total RNA $(100 \mathrm{ng})$ was detected using a NanoDrop ${ }^{\mathrm{TM}} 2000$ (Thermo Scientific ${ }^{T M}$ ) instrument. Complementary DNA was generated using the PrimeScript ${ }^{\circledR}$ RT reagent Kit with gDNA Eraser and
SYBR $^{\oplus}$ Premix DimerEraser ${ }^{\mathrm{TM}}$ (Takara Biotechnology Co., Ltd, Dalian, China). The gRT-PCR analysis was performed using SYBR Premix DimerEraser ${ }^{\top M}$ (Perfect Real Time) and LightCycler ${ }^{\oplus} 480$ (Roche Diagnostics, Penzberg, Germany). The annealing was performed at $60^{\circ} \mathrm{C}$ for $30 \mathrm{~s}$. Ultimately, relative gene expression was evaluated using the $2^{-\Delta \Delta \mathrm{Ct}}$ method with GAPDH as the internal control.

The following primers were used (forward $5^{\prime}-3^{\prime}$, reverse $5^{\prime}-3^{\prime}$ ): GAPDH, 5'-GTCATCCATGACAACTTTGG-3' and 5'-GAGCTTGA CAAAGTGGTCGT-3'; COL1A1， 5'-CTGGCAGCCCTGGTGAAAAT-3' and 5'-GGCAGCACCAGTAGCACC-3'; COL4A1, 5'-CCGGATCACATT GACATGAAACC- $3^{\prime}$ and $5^{\prime}$-CGAGAGGAACAATGCCTTGAG-3'.

\section{Western blot analysis}

Western blot analysis was conducted using standard protocols. HFL-1 cells were seeded on six-well plates and cultured for $24 \mathrm{~h}$. After a 3-day treatment with various concentrations of AA $(0,0.5$, 1.0 , or $2.0 \mu \mathrm{M})$, the cells were washed three times with ice-cold PBS and directly extracted into lysis buffer. The primary antibodies of COL1A1, COL4A1, and GAPDH (loading control) were used at a dilution ratio of $1: 1000$.

Immunofluorescence

HFL-1 cells were cultured in covered six-well glass-bottom plates for $24 \mathrm{~h}$ followed by 72 -h treatment with $\mathrm{AA}(0,0.5,1.0$, or $2.0 \mu \mathrm{M})$. The cells attached to the glass bottom were fixed with $4 \%$ formaldehyde at $4{ }^{\circ} \mathrm{C}$ for 20 min, permeabilized with PBS containing $0.2 \%$ Triton $\mathrm{X}-100$ for $20 \mathrm{~min}$, and blocked with $5 \% \mathrm{w} / \mathrm{v}$ bovine serum albumin (BSA) in PBS/T (PBS containing $0.1 \%$ Tween-20) at $37^{\circ} \mathrm{C}$ for $1 \mathrm{~h}$. An overnight incubation with primary antibodies of COL1A 1 and COL4A 1 at $4{ }^{\circ} \mathrm{C}$ followed by 1 -h treatment with fluorescence-conjugated secondary antibodies and 4',6-diamidino-2-phenylindole was performed before the cells were observed and imaged.

Xenografts and treatment

Female $\mathrm{BALB} / \mathrm{C}$ nude mice (weighing 16-22 g, animal license number SCXK (Shanghai) -2015000521727) obtained from Shanghai Laboratory Animal Center (Shanghai, China) were fed at the Center for Drug Safety Evaluation in Zhejiang University (Hangzhou, China, number of animal laboratory license, SYXK (Zhejiang) 2012-0178) and xenografted s.c. in the right flank with $1 \times 10^{7}$ SW620 cells. Once the tumor volumes reached approximately $100 \mathrm{~mm}^{3}$, the mice were randomly divided into five groups (five mice in each group) according to their tumor volumes on the day before treatment and assigned to treatment with saline (control group by intravenous injection), PLD (4 mg/kg on day $8,11,14$, and 17 by intravenous injection), AA ( $4 \mathrm{mg} / \mathrm{kg}$ once daily by i.g.), PLD (4 mg/kg) combined with low dosage of AA (LD-AA, $1 \mathrm{mg} / \mathrm{kg}$, i.g.) or high-dosage $A A(H D-A A, 4 \mathrm{mg} / \mathrm{kg}$, i.g.) at the same administration schedule of monotherapy (Fig. 1). Animal studies were conducted according to the National Research Council's guidelines and were approved by the Animal Experiment Ethics Committee of Zhejiang Cancer Hospital and Center for Drug Safety Evaluation of Zhejiang University (16031). All animals were sacrificed at day 18 . The tumor tissues were harvested, weighed, and subjected to evaluation of the intratumor concentration of DOX by in vivo fluorescence imaging. Each tissue sample was cut into two halves, one for quantitative analysis of DOX by highperformance liquid chromatography-mass spectrometry (HPLCMS/MS) and the other for collagen protein evaluation by Sirius $\mathrm{red} /$ fast green dual staining or immunohistochemistry (IHC) staining after embedding in paraffin.

\section{Expression of intratumor collagen proteins}

The collagen IV and total collagen levels in the tumor were evaluated by IHC staining and Sirius red/fast green dual staining, respectively. 

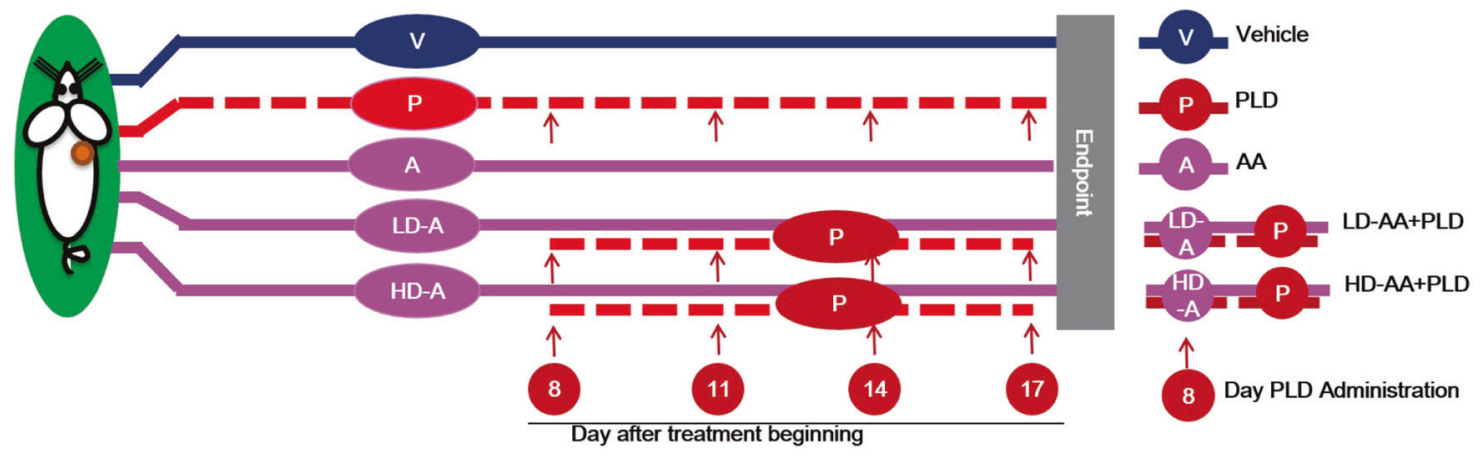

Fig. 1 Schedule of treatment

IHC staining. The expression of collagen type IV in the tumor was detected by IHC staining according to the reported method [23]. In brief, the de-paraffinized and de-hydrated paraffin tumor sections were treated with an initial 5-min blockage with $3 \%$ hydrogen peroxide followed by a 1-h blockage with 5\% BSA and PBS at $4{ }^{\circ} \mathrm{C}$, a $12-\mathrm{h}$ incubation with a primary antibody (AntiCollagen IV antibody, Abcam, USA. Ab6586), a 1-h incubation with a secondary antibody, a 15-min incubation with streptavidin peroxidase, and 1 min hematoxylin staining.

Sirius red/fast green dual staining. In brief, tumor sections (15 $\mu \mathrm{m}$ thick) were de-paraffinized, de-hydrated, and stained with Sirius red and fast green (30 min). The unbound dye was washed with redistilled water, which was removed by vigorous shaking. The bound dyes were extracted from the stained tissues with dye extraction solution and detected using a microplate reader (Bioteck ${ }^{\circledR}$, Vermont, USA) to evaluate the amounts of collagen and non-collagenous proteins based on $\mathrm{OD}_{540 \mathrm{~nm}}$ (for Sirius red) and $\mathrm{OD}_{605 \mathrm{~nm}}$ (for fast green), respectively. The ratios of collagenous to non-collagenous proteins in each section were calculated according to the manufacturer's instructions.

Estimation of DOX level within tumors

The intratumor exposure of DOX was evaluated by ex vivo fluorescence imaging and quantified by HPLC/MS analysis.

Ex vivo fluorescence Imaging. The harvested tumors were subjected to the Maestro Ex Vivo Imaging System (CRi, Woburn, MA, USA) for collection of fluorescence images of PLD. Two filter sets (blue: excitation at $500-720 \mathrm{~nm}$ with an exposure time of $300 \mathrm{~ms}$; red: excitation at $670-900 \mathrm{~nm}$ with an exposure time of $150 \mathrm{~ms}$ ) were used to detect the DOX-related fluorescence and tumor tissue-derived autofluorescence, respectively. The two sets of fluorescence images, the DOX-related fluorescence and autofluorescence spectra, were unmixed based on their spectral patterns using Maestro 2.6 software (CRi, Woburn, MA, USA).

HPLC-MS/MS analysis. The DOX distribution in the tumor tissues was analyzed by LC-MS/MS. Samples were pretreated and analyzed according to previously validated methods [24]. In brief, tumor tissues $(0.2 \mathrm{mg})$ were homogenized with $0.1 \%$ formic acid $(\mathrm{v} / \mathrm{v})$ at $4{ }^{\circ} \mathrm{C}$ to achieve a final homogenate containing $0.1 \mathrm{~g}$ tissue $/ \mathrm{mL}$. Homogenized tissue $(100 \mu \mathrm{L})$ was mixed with $400 \mu \mathrm{L}$ of ice-cold acetonitrile containing an internal standard $(125 \mathrm{ng} / \mathrm{mL}$ of daunorubicin) and vortexed for $3 \mathrm{~min}$. After centrifugation at $13000 \mathrm{rpm}$ and $4{ }^{\circ} \mathrm{C}$ for $10 \mathrm{~min}$, the supernatant was diluted with $0.1 \%$ formic acid solution $(1: 1, \mathrm{v} / \mathrm{v})$, and $20 \mu \mathrm{L}$ of mixture was injected into the LC-MS/MS for quantitative analysis.

Analysis was performed on an Agilent 1290/6460 LC-MS equipped with a triple quadrupole mass spectrometer using a Zorbax SB C8 column $(3.5 \mu \mathrm{m}, 150 \mathrm{~mm} \times 2.1 \mathrm{~mm})$. A $9 \mathrm{~min}$ gradient elution consisting of $0.1 \%$ formic acid solution $(\mathrm{v} / \mathrm{v})$ and acetonitrile $(15 \%$ at the first $0.5 \mathrm{~min}, 15-45 \%$ at $0.5-5.0 \mathrm{~min}$, $45-60 \%$ at $5.0-6.0 \mathrm{~min}, 60-15 \%$ at $6.0-6.5 \mathrm{~min}$, and $15-30 \%$ at the last $2.5 \mathrm{~min}$ ) was performed at a flow rate of $0.4 \mathrm{~mL} / \mathrm{min}$ at $35^{\circ} \mathrm{C}$. The MS analysis was performed using an electrospray ionization source in positive ion mode at the following settings: capillary voltage of $3000 \mathrm{~V}$; gas temperature of $325^{\circ} \mathrm{C}$; gas flow of $5 \mathrm{~L} / \mathrm{min}$; nebulizing pressure of $45 \mathrm{psi}$; sheath gas temperature of $350^{\circ} \mathrm{C}$; and sheath gas flow of $11 \mathrm{~L} / \mathrm{min}$. Multiple reaction monitoring scan mode monitored the ion pair of DOX at $\mathrm{m} / \mathrm{z} 544.0 \rightarrow 361.0$ and $544.0 \rightarrow 397.1$ and daunorubicin at $\mathrm{m} / \mathrm{z} 528.0 \rightarrow 321.0$ and $528.0 \rightarrow 363.1$.

\section{Antitumor effect in vivo}

The length and width of the tumor were measured twice every week using a caliper. The volume $\left(\mathrm{TV}_{n}, \mathrm{~mm}^{3}\right)$ and relative volume $\left(\operatorname{RTV}_{n}, \%\right)$ of the tumor at day $n$ were calculated according to the formulas $\mathrm{TV}_{n}=\left[\right.$ length $\times$ width $\left.{ }^{2}\right] / 2$ and $\mathrm{RTV}=\mathrm{TV}_{n} / \mathrm{TV}_{0}\left(\mathrm{TV}_{0}\right.$, tumor volume at day 0$)$, respectively. The bodyweights of mice were monitored daily and followed as a general index of systemic toxicity. The inhibition ratio (IR, \%) of the tumor volumes was used as the index of the antitumor effect, which was calculated as follows: IR $(\%)=\left(1-\mathrm{RTV}_{\text {treated }} / \mathrm{RTV}_{\text {control }}\right) \times 100 \%$, where $\mathrm{RTV}_{\text {treated }}$ and $\mathrm{RTV}_{\text {control }}$ represent the average tumor volumes of the treatment group and control group, respectively.

\section{Statistical analysis}

Student's $t$-test, linear regression, and the repeated measure test were appropriately applied. The profile of the tumor growth during the study was examined using the repeated measure test. The level of $P<0.05$ was accepted to determine statistical significance. All calculations were performed using SPSS Statistics 17.0 (SPSS Inc., Dublin, Ireland) and Graphpad Prism 7 (GraphPad Software, La Jolla, California, USA).

\section{RESULTS}

AA improves the in vivo antitumor activity of PLD

Combination therapy with AA achieved an increased inhibition rate of tumor growth $(\mathrm{IR}=57.3 \%)$, significantly greater than that of AA (IR $=5.5 \%, P<0.001)$ or PLD (IR $=35.9 \%, P=0.043$, Fig. $2 \mathrm{a}$, c) alone. Similarly, significant tumor shrinkage (44\% reduction in tumor weight) was observed in the HD-AA + PLD group compared with the mono-PLD group $(0.84 \pm 0.22$ vs. $1.50 \pm 0.16 \mathrm{~g}, P=0.043$, Fig. 2c), although the shrinkage was not significant in the LD-AA + PLD arm $(1.18 \pm 0.10$ vs. $1.50 \pm 0.16 \mathrm{~g}, P=0.262)$. The relative weight of mice varied from to 96.7 to $105.4 \%$ in various groups (Fig. 2d). In addition, no significant improvement was noted in the cytotoxic effect of PLD or free DOX when combined with $A A$ in vitro (Fig. 2e, f). The half maximal inhibitory concentration $\left(\mathrm{IC}_{50}\right)$ of AA combined PLD $\left(\mathrm{IC}_{50}: 1.05,1.75\right.$, and $1.88 \mu \mathrm{M}$ at $\mathrm{AA}$ doses of $0.5,1.0$, and $2.0 \mu \mathrm{M})$ or DOX $\left(\mathrm{IC}_{50}: 0.41\right.$, 0.47 , and $0.46 \mu \mathrm{M}$ at $\mathrm{AA}$ doses of $0.5,1.0$, and $2.0 \mu \mathrm{M}$ ) were 


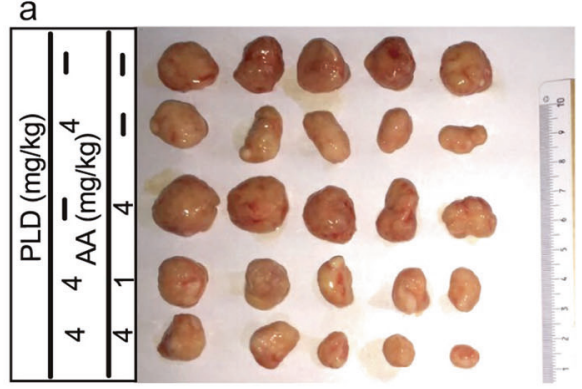

b
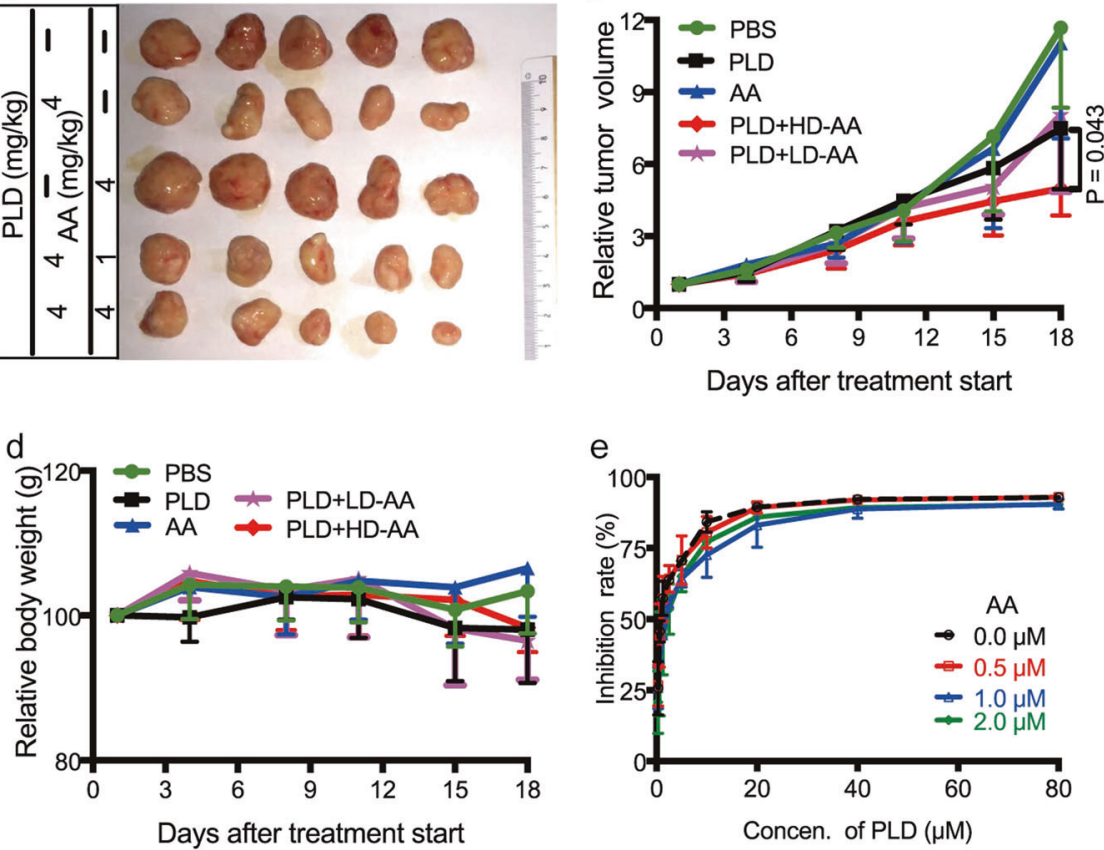

e

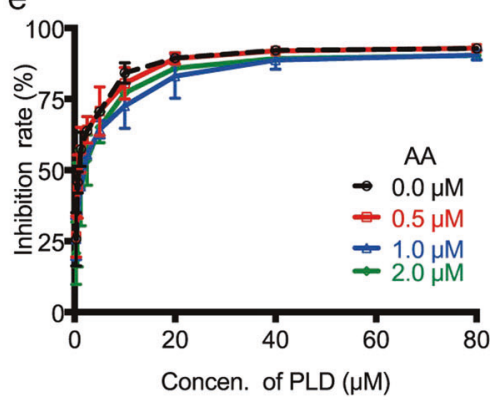

C

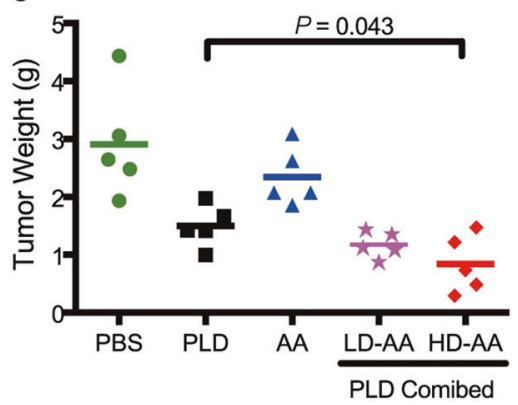

f

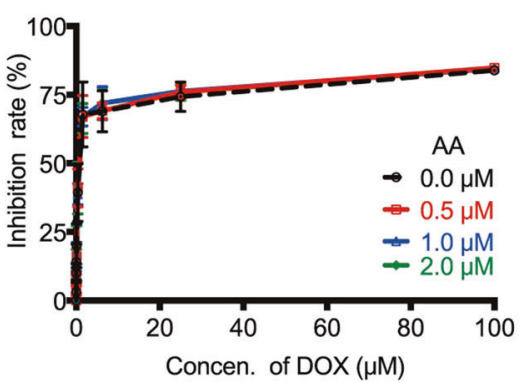

Fig. 2 Antitumor effect of combined treatment with PLD and AA in SW620 xenograft nude mice and SW620 cell line. In vivo, the combined regime of PLD and AA displayed an enhanced antitumor effect with smaller tumor volume (a, b), lower tumor weight (c), and comparable bodyweight (d) compared with PLD $(4 \mathrm{mg} / \mathrm{kg})$ alone. However, in vitro, no significant improvement was observed in the cytotoxic effect of PLD (e) and DOX (f) by AA
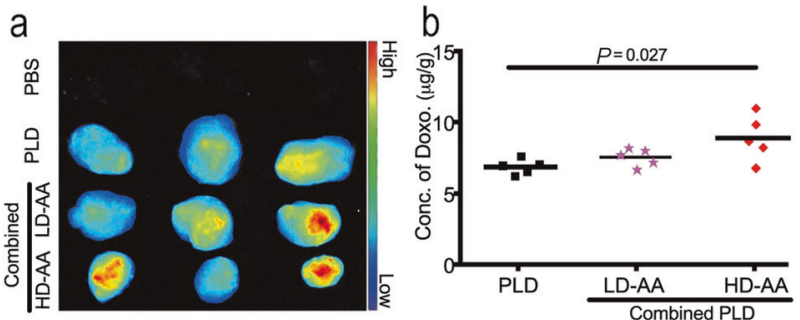

Fig. 3 Doxorubicin accumulation in SW620 xenograft tumor. (a) Ex vivo fluorescence imaging. The intensity of doxorubicin in the HDAA combined group was significantly increased compared with mono-PLD treatment. (b) Concentration of doxorubicin quantified by HPLC-MS/MS. The intratumor level of doxorubicin was significantly increased by combination with high-dosage AA ( $4 \mathrm{mg} / \mathrm{kg}$, red diamond) compared with mono-PLD treatment (black square, $P=0.005)$, whereas low-dosage AA (1 mg/ $\mathrm{kg}$, pink star) failed to enhance intratumor exposure of doxorubicin

comparable or even higher than that of PLD alone $\left(\mathrm{IC}_{50}=1.02 \mu \mathrm{M}\right)$ or DOX alone $\left(\mathrm{IC}_{50}=0.43 \mu \mathrm{M}\right)$.

AA acutely increases deposition of DOX in SW620 xenograft tumors

Figure 3 shows an image of intratumor DOX. The fluorescence intensity of intratumor DOX was significantly elevated by AA pretreatment, particularly HD-AA, which induced a nearly $30 \%$ elevation compared with PLD alone $(8.88 \pm 0.72$ vs. $6.86 \pm$ $0.23 \mu \mathrm{g} / \mathrm{g}, P=0.027$ ). In contrast, the DOX level in the LD-AA combination group was $7.53 \pm 0.28 \mu \mathrm{g} / \mathrm{g}$, closer to that of the mono-PLD treatment group $(P=0.098)$.

AA reduces intratumor deposition of collagenous proteins The fraction of collagen IV proteins in paraffin-embedded tumor sections was visualized by IHC staining and hematoxylin and eosin staining (Fig. 4a). A significant shrinkage of the stromal region and a reduction in collagen IV deposition were observed in the presence of $A A$. The relative fraction of collagen in mono-PLDtreated tumors was significantly reduced by $22 \%$ (from $4.14 \% \pm$ $0.30 \%$ to $3.24 \% \pm 0.25 \%, P=0.051$ ) with HD-AA or by $11 \%$ (to $3.69 \% \pm 0.27 \%, P=0.133$ ) with LD-AA. In contrast, no significant differences were observed in the amount of collagen deposited between the samples treated with PBS and PLD $(4.41 \% \pm 0.33 \%$ of PBS and $4.14 \% \pm 0.30 \%$ of PLD, $P=0.576$, Fig. $4 \mathrm{~b}$ ). The intratumor level of DOX was found to be related to the total collagen level $(r=0.651, P=0.009$, Fig. $4 c)$.

AA inhibits fibroblast-derived collagenous protein levels Compared with AA-free $C M$, the mRNA levels of collagen I $(\mathrm{COL} 1 \mathrm{~A} 1)$ and IV $(\mathrm{COL} 4 \mathrm{~A} 1)$ were significantly reduced $(P<0.0001)$ by $66.4 \% \pm 7.6 \%$ to $87.4 \% \pm 3.9 \%$ and $71.7 \% \pm 4.6 \%$ to $87.2 \% \pm$ $1.3 \%$, respectively (Fig. 5a). A significant attenuation of collagenous proteins was observed in the western blot analysis (Fig. 5b) and IHC staining (Fig. 5c).

\section{DISCUSSION}

PLD was the first long-circulation liposome approved by the Food and Drug Administration for treatment of AIDS-related kaposi sarcoma, multiple myeloma, and advanced ovarian cancer. Paradoxically, the clinical benefit of PLD is not superior to that of conventional DOX in various solid tumors [3]. The meaningfully restricted delivery of a vector from the blood vessels to cancer cells induced by the dense stroma network was considered a potential contributor to the compromised effect [25], especially for large-size nanoparticles (diameter $>50 \mathrm{~nm}$ ) [26]. Therefore, alleviation of the stroma was proposed as a novel strategy to boost the clinical benefit of DDSs.

In the current study, AA treatment was conducted to alleviate the stroma level and facilitate penetration of PLD from the microvessels to the cancer cells to boost its efficacy. The results showed that HD-AA enhanced the in vivo response to four cycles 
a
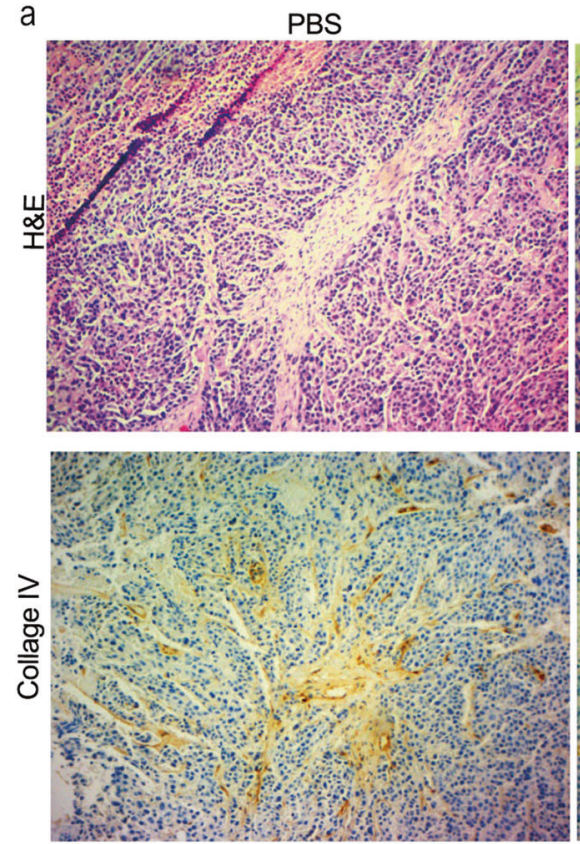

AA
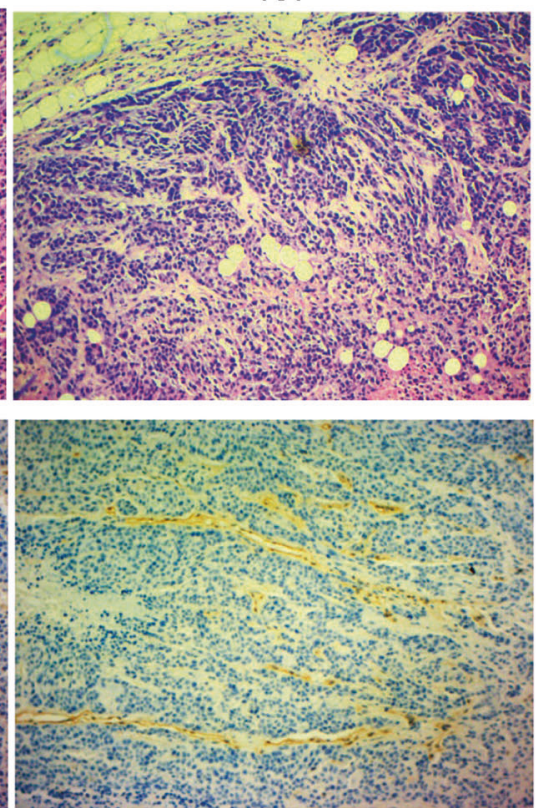

b
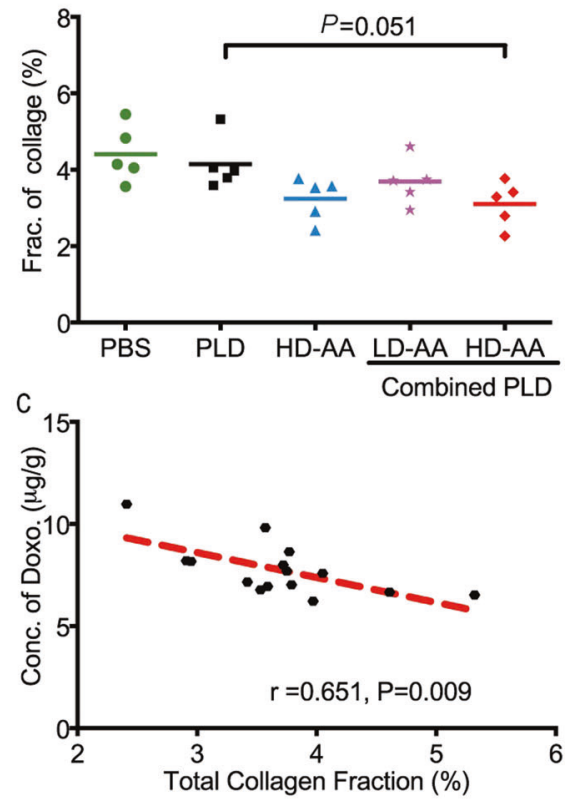

Fig. 4 Intratumor-stroma alleviation by AA in SW620 xenograft mouse models. (a) H\&E staining and IHC staining of tumor sections treated with PBS and AA $(4 \mathrm{mg} / \mathrm{kg})$. The fraction of stroma and collagen IV was reduced in the AA-treated group compared with the PLD-treated group. (b) Relative fraction of total collagen proteins in tumor tissue evaluated using the semi-quantitative kit. AA significantly reduced the level of collagen IV. (c) A correlation between the intratumor level of doxorubicin and total collagen was observed $(r=0.663, P=0.007)$

a

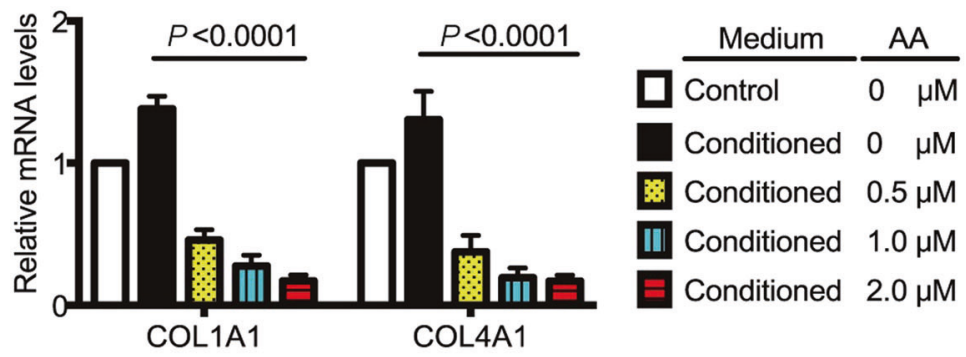

b

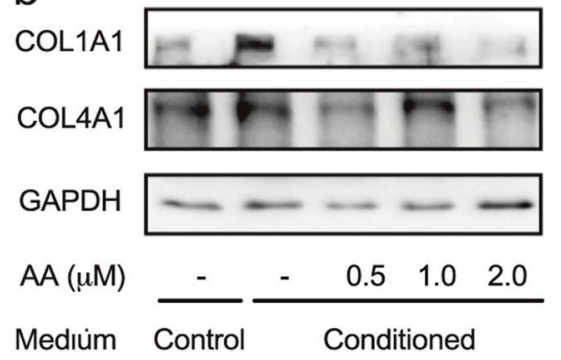

C
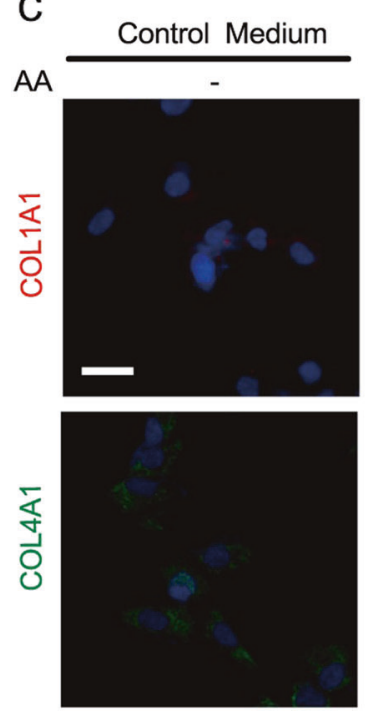

Conditioned Medium
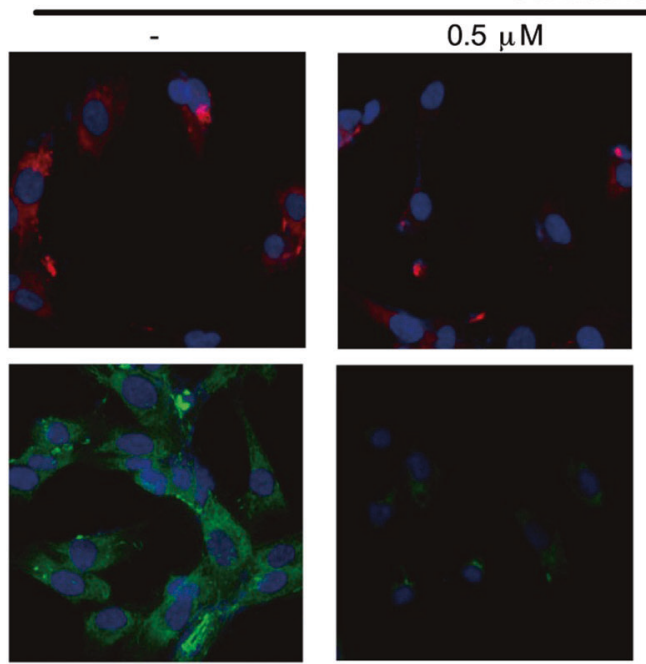

$1.0 \mu \mathrm{M}$
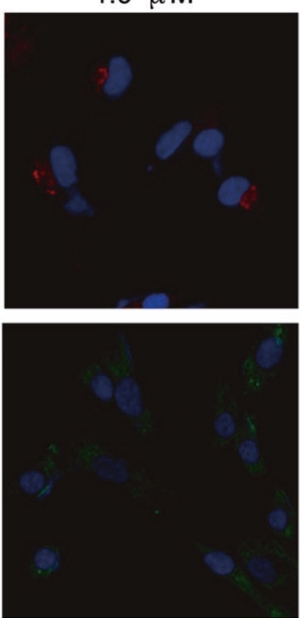
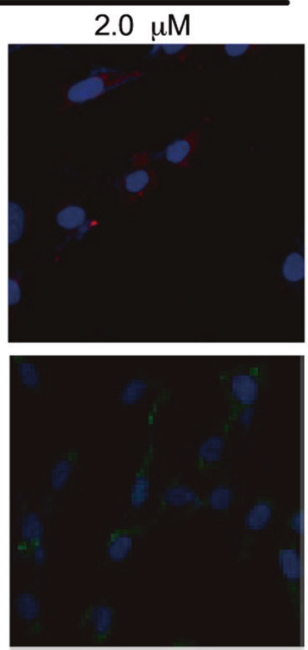

Fig. 5 AA reversed the increased mRNA and protein levels of COL1A1 and COL4A1 in HFL-1 cells induced by SW620-derived conditioned medium. The mRNA levels of COL1A1 and COL4A1 were analyzed using fluorescent quantitative RT-PCR (a). Error bars represent standard deviations (SDs). Type I and IV collagen proteins were detected by Western blotting (b) and visualized by immunofluorescence (c). Scale bar, $25 \mu \mathrm{m}$ 
of PLD with $\sim 60 \%$ elevation of the tumor inhibition rate. To the best of our knowledge, losartan was the only agent reported to improve the antitumor effect of PLD but only in a pilot study for PLD. Although $50 \%$ tumor shrinkage was observed by combination with losartan, detailed information, including intratumor exposure improvement of PLD and its correlation with collagen level, was not reported [16]. In addition, a comparable effect of AA and PBS on tumor growth inhibition was observed in our study. Moreover, no significant synergy of $A A$ and DOX was observed in the cytotoxic effect. In contrast, the $I C_{50}$ values of $A A$ combined with PLD or DOX were comparable to those of PLD or DOX administration alone.

The synergetic effect might be attributed to the stromaremodeling effect of $A A$. According to our findings, $A A$ significantly reduced the intratumor deposition of collagen by $22 \%$ in vivo. AA also significantly inhibited the activation of fibroblasts induced by cancer cells and reduced the collagenous levels (mRNA levels reduced by $~ 90 \%$ ) in vitro. Two methods of stroma modulation with potential translational potency were reported by Diop-Frimpong et al. [16] and Olive et al. [17]. However, only Diop-Frimpong reported a quantitative effect of collagen alleviation in vivo in which approximately $20-65 \%$ of collagen I was eliminated in xenografts by losartan $(60 \mathrm{mg} / \mathrm{kg} /$ day $)$ [16]. The efficiency of AA disclosed in our study was comparable. AA serves broad-spectrum functions of anti-fibrosis in the liver, kidney, and skin via inhibition of collagen matrix expression by fibroblasts $[19,20,27,28]$. A wide range of doses from 0.5 to $16 \mathrm{mg} / \mathrm{kg}$ was adopted $[19,27,28]$. However, considering that AA was found to be a potential anti-neoplastic agent at high doses $(\geq 30 \mathrm{mg} / \mathrm{kg} /$ day $[29,30])$ and that our objective was simply to estimate its stroma-remodeling effect on the PLD efficacy, it was preferably administered at low doses to avoid the potentially direct antitumor effect of high-dose AA in the current study. The antifibrotic effect in the tumor might be associated with its general mechanism of action via improved Smad7 expression, activation of PPAR- $\gamma$, or blockage of the phosphorylation of p38 MAPK and ERK1/2 of fibroblasts $[19,20]$. However, the specific mechanisms involved in tumor-stroma remodeling must be further disclosed.

A reasonable approach to increasing the antitumor efficacy of DOX is to elevate its intratumor level. According to our findings, the intratumor concentration of DOX was $6.86 \mu \mathrm{g} / \mathrm{g}$, comparable to previous reports of bone metastatic breast carcinoma that received PLD with a concentration of $6.5 \mu \mathrm{g} / \mathrm{g}$ at a dose of $50 \mathrm{mg} / \mathrm{m}^{2}$ and $1.4 \mu \mathrm{g} / \mathrm{g}$ at a dose of $35 \mathrm{mg} / \mathrm{m}^{2}$ [31]. An adverse correlation was found between the total collagen fraction and the intratumor DOX level $(r=0.651, P=0.009)$. The AAinduced reduction in collagen elevated the DOX level by $30 \%$ $(8.88 \mu \mathrm{g} / \mathrm{g})$. As reported, the inhibition of stromal collagen could lead to increased delivery of nanoparticles (nanoparticles $100 \mathrm{~nm}$ in diameter) by 1.5 -fold in HSTS26T and 4-fold in Mu89 [17].

Although our study confirmed the clinical benefit of stroma remodeling, its limits should also be mentioned. AA enhanced the antitumor effect of PLD to a significantly higher but less than optimal level. This result could be partially attributed to a stromalimited microenvironment of the cell line-derived xenograft. Therefore, a stroma-rich animal model such as a patient-derived xenograft might serve as a better model for further research. Moreover, we can only state that "AA enhances the intratumor delivery and antitumor effect of PLD by attenuating tumor-stroma collagens" can be addressed. It was difficult to determine whether this effect is a Tt-DDS-specific effect or not due to the absence of data from the "AA + DOX" group. Therefore, a further study will be conducted to confirm whether the impact of AA-induced stroma remodeling on anti-neoplastic agents is Tt-DDS-specific or not. Additionally, all animals were sacrificed on the day after the last PLD administration to obtain tumor tissue for determination of the intratumor levels of PLD in the presented paper. Therefore, the impact of AA on the survival outcome of PLD in animals was not evaluated in this study.

\section{CONCLUSION}

The dense matrix in the tumor-stroma was identified as one of negative factors for patient benefits from Tt-DDSs. The current study investigated the role of $A A$ in reducing the stroma to improve the antitumor effect of PLD. Pretreatment with AA induced a significant reduction in stromal collagens and increased accumulation of PLD in the tumor, with an improved in vivo effect of PLD. In conclusion, the study revealed that AA is an efficient agent for tumor-stroma remodeling and chemotherapy sensitization, thus offering a potential strategy to sensitize chemotherapy for cancer care.

\section{ACKNOWLEDGEMENTS}

The authors thank Dr. Qin-jie Weng and Dr. Xiao-yang Dai at the Zhejiang Province Key Laboratory of Anti-Cancer Drug Research, College of Pharmaceutical Sciences, Zhejiang University, China, for xenograft-model building; Dr. Xiao-dan Wu and Dr. Zhi-wei Ge at the Center of Analysis and Measurement, Zhejiang University, China, for HPLC-MS/MS analysis of doxorubicin; and Dr. Tian-yuan Zhang at the Institute of Pharmaceutics, Zhejiang University, China, for ex vivo fluorescence imaging of doxorubicin. This work was supported by the National Natural Science Foundation of China (81301873 and 81773819), the Natural Science Foundation of Zhejiang Province (LY16H160038 and LQ17H300001), the Science and Technology in Zhejiang Province Chinese Medicine Program (2015ZA036), the Scientific Research Key Program of the Health Bureau of Zhejiang Province (WKJ-ZJ-1504), the Medical Science Research Foundation of Zhejiang Province (2015ZDA006), the Zhejiang Provincial Program for the Cultivation of 151 Talents (PH and LF), the Zhejiang Provincial Program for the Cultivation of High-level Innovative Heath Talents (Ping Huang), and the Zhejiang Cancer Hospital Program for the Cultivation of 1022 Talents (LF).

\section{AUTHOR CONTRIBUTIONS}

F-ZL, PH, and LF designed the research. LF, S-sK, L-kZ, Y-jL, C-mW, and JS performed the research. LF, S-sK, and $H-y D$ analyzed the data. LF, Y-jL, and $Y-w Z$ wrote the paper. All authors approved the final manuscript.

\section{ADDITIONAL INFORMATION}

Competing interests: The authors declare no competing interests.

\section{REFERENCES}

1. van Elk M, Murphy BP, Eufrasio-da-Silva T, O'Reilly DP, Vermonden T, Hennink WE, et al. Nanomedicines for advanced cancer treatments: transitioning towards responsive systems. Int J Pharm. 2016;515:132-64.

2. Petersen GH, Alzghari SK, Chee W, Sankari SS, La-Beck NM. Meta-analysis of clinical and preclinical studies comparing the anticancer efficacy of liposomal versus conventional non-liposomal doxorubicin. J Control Release. 2016;232:255-64.

3. Gabizon AA, Patil Y, La-Beck NM. New insights and evolving role of pegylated liposomal doxorubicin in cancer therapy. Drug Resist Updat. 2016;29:90-106.

4. Gabizon A, Shmeeda H, Grenader T. Pharmacological basis of pegylated liposomal doxorubicin: impact on cancer therapy. Eur J Pharm Sci. 2012;45:388-98.

5. Ramanujan S, Pluen A, McKee TD, Brown EB, Boucher Y, Jain RK. Diffusion and convection in collagen gels: implications for transport in the tumor interstitium. Biophys J. 2002;83:1650-60.

6. Netti PA, Berk DA, Swartz MA, Grodzinsky AJ, Jain RK. Role of extracellular matrix assembly in interstitial transport in solid tumors. Cancer Res. 2000;60:2497-503.

7. Dellian M, Yuan F, Trubetskoy VS, Torchilin VP, Jain RK. Vascular permeability in a human tumour xenograft: molecular charge dependence. $\mathrm{Br} J$ Cancer. 2000;82:1513-8.

8. Sato H, Takino T, Okada Y, Cao J, Shinagawa A, Yamamoto E, et al. A matrix metalloproteinase expressed on the surface of invasive tumour cells. Nature. 1994;370:61-65.

9. Jain RK, Stylianopoulos T. Delivering nanomedicine to solid tumors. Nat Rev Clin Oncol. 2010;7:653-64. 
10. Miao L, Huang L. Exploring the tumor microenvironment with nanoparticles. Cancer Treat Res. 2015;166:193-226.

11. Kim JH, Lee YS, Kim H, Huang JH, Yoon AR, Yun CO. Relaxin expression from tumor-targeting adenoviruses and its intratumoral spread, apoptosis induction, and efficacy. J Natl Cancer Inst. 2006;98:1482-93.

12. Ganesh S, Gonzalez Edick M, Idamakanti N, Abramova M, Vanroey M, Robinson M, et al. Relaxin-expressing, fiber chimeric oncolytic adenovirus prolongs survival of tumor-bearing mice. Cancer Res. 2007;67:4399-407.

13. Mok W, Boucher Y, Jain RK. Matrix metalloproteinases-1 and -8 improve the distribution and efficacy of an oncolytic virus. Cancer Res. 2007;67:10664-8.

14. Eikenes L, Tari M, Tufto I, Bruland OS, de Lange Davies C. Hyaluronidase induces a transcapillary pressure gradient and improves the distribution and uptake of liposomal doxorubicin (Caelyx) in human osteosarcoma xenografts. Br J Cancer. 2005;93:81-88.

15. McKee TD, Grandi P, Mok W, Alexandrakis G, Insin N, Zimmer JP, et al. Degradation of fibrillar collagen in a human melanoma xenograft improves the efficacy of an oncolytic herpes simplex virus vector. Cancer Res. 2006;66:2509-13.

16. Diop-Frimpong B, Chauhan VP, Krane S, Boucher Y, Jain RK. Losartan inhibits collagen I synthesis and improves the distribution and efficacy of nanotherapeutics in tumors. Proc Natl Acad Sci USA. 2011;108:2909-14.

17. Olive KP, Jacobetz MA, Davidson CJ, Gopinathan A, Mclntyre D, Honess D, et al. Inhibition of Hedgehog signaling enhances delivery of chemotherapy in a mouse model of pancreatic cancer. Science. 2009;324:1457-61.

18. Adtani PN, Narasimhan M, Punnoose AM, Kambalachenu HR. Antifibrotic effect of Centella asiatica Linn and asiatic acid on arecoline-induced fibrosis in human buccal fibroblasts. J Investig Clin Dent. 2017;8. https://doi.org/10.1111/jicd.12208. Epub 2016 Feb.

19. Tang LX, He RH, Yang G, Tan JJ, Zhou L, Meng XM, et al. Asiatic acid inhibits liver fibrosis by blocking TGF-beta/Smad signaling in vivo and in vitro. PLoS ONE. 2012;7:e31350.

20. Bian D, Zhang J, Wu X, Dou Y, Yang Y, Tan Q, et al. Asiatic acid isolated from Centella asiatica inhibits TGF-beta1-induced collagen expression in human keloid fibroblasts via PPAR-gamma activation. Int J Biol Sci. 2013;9:1032-42.
21. Huo L, Shi W, Chong L, Wang J, Zhang K, Li Y. Asiatic acid inhibits left ventricular remodeling and improves cardiac function in a rat model of myocardial infarction. Exp Ther Med. 2016;11:57-64.

22. Ramachandran V, Saravanan R, Senthilraja P. Antidiabetic and antihyperlipidemic activity of asiatic acid in diabetic rats, role of HMG COA: in vivo and in silico approaches. Phytomedicine. 2014;21:225-32.

23. Wang J, Lu L, Wang Y, Wu Y, Han J, Wang W, et al. Qishenyiqi Dropping Pill attenuates myocardial fibrosis in rats by inhibiting RAAS-mediated arachidonic acid inflammation. J Ethnopharmacol. 2015;176:375-84.

24. Baumgarten S, Gaba RC, van Breemen RB. Confirmation of drug delivery after liver chemoembolization: direct tissue doxorubicin measurement by UHPLC-MSMS. Biomed Chromatogr. 2012;26:1529-33.

25. Miao L, Lin CM, Huang L. Stromal barriers and strategies for the delivery of nanomedicine to desmoplastic tumors. J Control Release. 2015;219: 192-204.

26. Dhanikula RS, Argaw A, Bouchard JF, Hildgen P. Methotrexate loaded polyethercopolyester dendrimers for the treatment of gliomas: enhanced efficacy and intratumoral transport capability. Mol Pharm. 2008;5:105-16.

27. Xu C, Wang W, Xu M, Zhang J. Asiatic acid ameliorates tubulointerstitial fibrosis in mice with ureteral obstruction. Exp Ther Med. 2013;6:731-6.

28. Wu F, Bian D, Xia Y, Gong Z, Tan Q, Chen J, et al. Identification of major active ingredients responsible for burn wound healing of Centella asiatica herbs. Evid Based Complement Altern Med. 2012;2012:848093.

29. Kavitha CV, Jain AK, Agarwal C, Pierce A, Keating A, Huber KM, et al. Asiatic acid induces endoplasmic reticulum stress and apoptotic death in glioblastoma multiforme cells both in vitro and in vivo. Mol Carcinog. 2015; 54:1417-29.

30. Wu T, Geng J, Guo W, Gao J, Zhu X. Asiatic acid inhibits lung cancer cell growth in vitro and in vivo by destroying mitochondria. Acta Pharm Sin B. 2017;7:65-72.

31. Symon Z, Peyser A, Tzemach D, Lyass O, Sucher E, Shezen E, et al. Selective delivery of doxorubicin to patients with breast carcinoma metastases by stealth liposomes. Cancer. 1999;86:72-8. 